\title{
A NEW APPROACH FOR NAVIGATION AND TRAFFIC SIGNS INDICATION USING MAP INTEGRATED AUGMENTED REALITY FOR SELF-DRIVING CARS
}

\author{
HARSHAL DEORE*, AKSHAT AGRAWAL* † VIVEK JAGLAN $\ddagger$ POOJA NAGPAL§ AND MAYANK MOHAN SHARMA
}

\begin{abstract}
Self-driving vehicles are one of the emerging technologies. This technology has potential to save lives and make lives comfortable. However, the technology used in self driving cars has to perform series of task for building perceptions. This has some certain prerequisites related to road infrastructure and is affected by daylight and weather conditions of the place. If these prerequisites are not satisfied then it could affect the performance of the vehicle and can be considered as compromise with safety of the users. This research work is focused on trying to find a new approach using which the underdeveloped countries will also be able to implement self driving cars in their county. The objective of this paper is to propose a new approach to supplement the technology used in the self-driving cars for perception. Using this approach the countries who don't satisfy the prerequisites would be eligible to implement them without compromising the safety.

The proposed approach uses the technology Augmented Reality to create and augment artificial objects of navigational signs and traffic signals based on vehicles location to reality. Later the augmented scene is fed into the conventional Deep learning object detection algorithm to detect the navigational artificial objected along with other real objects. This approach help navigate the vehicle even if the road infrastructure does not have very good sign indications and marking.

The approach was tested locally by creating a local navigational system and a smartphone based augmented reality app. The approach performed better than the conventional method as the objects were clearer in the frame which made it each for the object detection to detect them.
\end{abstract}

Key words: Self Driving Cars, Augment Reality,Machine Vision

AMS subject classifications. 68T40,68T45

1. Introduction. Every year huge amount of money is spent on research for finding cures and vaccines, but people often fail to recognize magnitude and cost of road traffic accidents. Each year $2.2 \%$ of deaths are caused by road traffic accidents this makes it 9th leading cause of death in the world. Nearly 12.5 lakh people each year which mean on an average 3287 people die daily on the road due to road traffic accidents. Addition to that every year 2-5 crores of people are left injured. In these deaths the people aging between 15-44 account for more than 50\%. Surprisingly, among 15-29 years old it is leading cause of death, and among 5-14 years old it is second leading cause of death globally. The number of people below age 25 dying in road traffic accidents is around 4 lakhs, which makes it 1000 young people each day. More than $90 \%$ of these deaths occur in countries which are underdeveloped or have low income. These accidents don't only cause fatalities but harm the economy also, It has been found that these accidents costs $\$ 518$ worldwide, on an average 1 to 2 percent of an individual countries GDP. This expense is more than the amount few underdeveloped countries receive for development assistance [1].

Innovation has improved technology to a great extent over past few decades. The technologies have disrupted various industries for betterment of the society. One such innovation in the field of Artificial Intelligence was Deep Learning. Deep learning has enabled us to bring human like or even better perception in machines. The technology is set to disrupt various industries one such industry is transport. Innovation of deep learning has led people to build self-driving cars. Self-driving cars concept is to replace need of person to drive them from one place to another. These vehicles have cameras mounted over them which continuously capture the

\footnotetext{
* Amity University Haryana (harshaldeore7@gmail.com).

$\dagger$ Amity University Haryana(akshatag20@gmail.com).

$\ddagger$ Graphic Era Hill University(jaglanvivek@gmail.com).

$\S$ Amity University Haryana(pbnagpal@ggn.amity.edu).

ๆZillow Inc., San Francisco, U.S.A.(mayank.mohan.sharma@gmail.com).
} 
surrounding and is fed into the deep learning algorithm which detects if there is any object which could cause an accident. The vehicle's control system totally depends on the detection algorithm and responds based on its findings. Self-driving vehicles are currently need human driver presence just work as an assistant as it is not yet fully developed. These vehicles are improving day by day and will be there in each and every corner of the world in the coming years.

Considering the statistics of deaths and injuries caused by road accidents it is evident that self- driving vehicle (self driving vehicles) are the future of transport. Around 47 cities in the world are piloting self driving vehicles projects. A report prepared by KPMG shows Autonomous Vehicles Readiness Index (AVRI) for 25 countries. The AVRI is a tool to measure how well a country is prepared to implement self driving vehicles, this tool considers 25 different factors and all these factors are scored in a single digit. It is quite clear that to successfully deploy self driving vehicles in a country, the country must have good road infrastructure. The good infrastructure for self driving vehicles means the lane marking should be proper, the traffic signals and sign boards should be present at all the essential locations. The lack of infrastructure has been the main reason behind the lag in implementation of this life saving technology [2].

The self driving vehicles have multiple sensor and cameras mounted on and around it. These cameras capture the surrounding and those images are fed into Deep learning $D L$ algorithms to detect and recognize various objects. The results get affected with quality of cameras, surrounding condition like sunlight falling at low angle or it is too bright causes difficulty in reading traffic lights. Also fog, smog, snowfall make it difficult to work. Deep learning algorithm depends on data, providing it with more data with different scenarios its results improve. Hence it is very important it gets implemented in more countries and different places. This will help in collecting more data and gradually lead to better and safer self driving vehicles [3].

Countries like India are in the most need of self driving vehicles. In India, there were 464,674 road accidents which caused 148,707 deaths. In the population of 1.31 billion there are 182.45 million vehicles. In 2015 the collision rate was 0.8 per 1000 vehicles and per 1000,000 people there were 11.35 deaths [4].

Poor road infrastructure and poor surrounding conditions are the two problems which create a question mark over the performance and safety of self driving vehicles. The approach proposed in this paper has aim to help these self-driving vehicles reach the countries where they are most needed. The approach uses augmented reality technique to supplement the conventional method.

\section{Objectives:}

- Developing an algorithm to combine deep learning and augmented reality technologies

- Creating test setup to test the proposed approach

- Comparing results of the proposed approach

The paper is spread in four sections. Section 2 discusses related work followed by methodology in section 3. Section 4 presents results and comparison and finally section 5 discusses conclusion of current study with future directions.

\section{Related Works.}

2.1. Augmented Reality. Virtual Reality $V R$ is used to create a virtual world with the use of computer graphics which can experienced through wearable devices. Telepresence is also same kind of technology used to facilitate someone's problem solving skills from a remote place [5].

However, Augmented Reality $A R$ is another technology which is often referred as Mixed Reality $M R$ as how it works is combination of both the VR and Telepresence technology.

In AR, a layer of artificial objects created by us to the real-world perception is added, these artificial can be considered as extra information to make the real world more informative. This extra information or the layer of the artificial objects does not cover up any important or meaning detail from the real-world but enriches the experience. In simple words AR is VR with added real world. The technology is being used widely to help students learn topics practically which are otherwise difficult to image. AR make learning interesting and joyful as students get to see virtual objects in real-life environment [6].

AR was invented with a desire to make build airplanes easier. In 1990, Caudell and Mizell developed a system for workers to help them during assembly for guiding them they could wear the device on their heads. The device would overlay real world with graphical instructions [7]. 


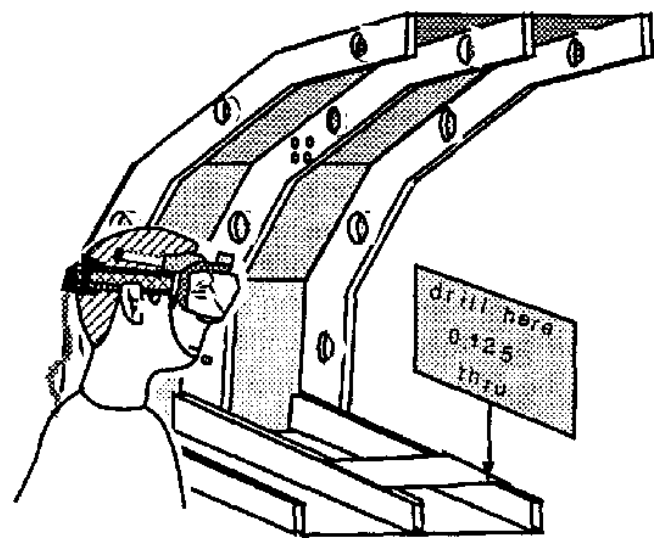

FIG. 2.1. AR guiding worker for drilling [7]

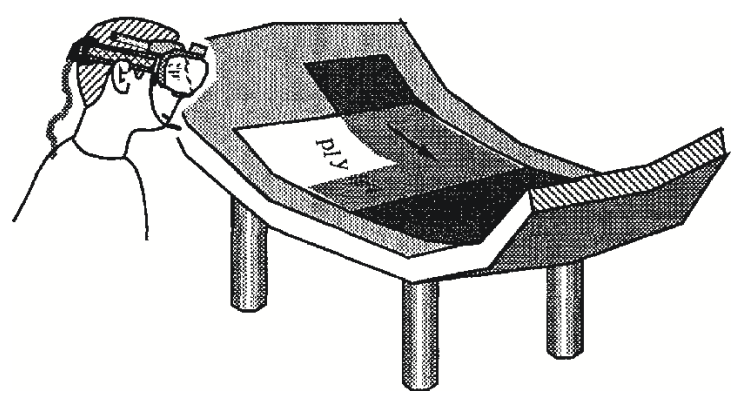

FIG. 2.2. AR guiding worker with ply [7]

However, the initial attempts were not successful as the system could not track head positions efficiently and was not responsive enough when people moved around. The main reason behind that was the computer inside the wearable was not powerful enough back then.

Furthermore, the idea was not limited to industrial applications. In 2000, Bruce Thomas along with other researchers developed an extended version of a desktop game called Quake. They converted that first-person desktop game to outdoor/indoor mobile AR game [8].

Similarly, it can be used for navigation purpose and people have been putting efforts in creating mobile based applications to help foreign people navigate at new place like Airport, Hotels or offices [9].

2.2. Deep Learning. Deep learning's birth came from people's aspiration to create machines which could act, think and behave like humans. In 1943, McCulloch and Pitts studied functioning of neurons inside the brain and tried to understand how they could produce complex patterns with a system of tiny cell connected to each other. They then created a small simplified model of neuron. This model had limitations but made an important contribution to development of artificial neural networks. Later they could add learning feature to the model [10].

Furthermore, in 1958 Frank Rosenblatt proposed the concept of perceptron this is considered as major development in neural networks. In his model he first passed the input through some pre-processors also called association units. Pre-processors work was to detect if there are any specific features present in the inputs [11].

However, in 1969 Minsky along with Seymour Papert attacked the work of Frank Rosenblatt where they introduced the XOR problem. They proved that a single perceptron a grandparent to the computational units which compose modern neural networks was incapable of learning the exclusive-or (aka XOR) function [12].

As a result, in 1986 David E. Rumelhart along with two other researchers introduced a new learning procedure for neural networks. The aim of the procedure was to minimize the difference between actual output 


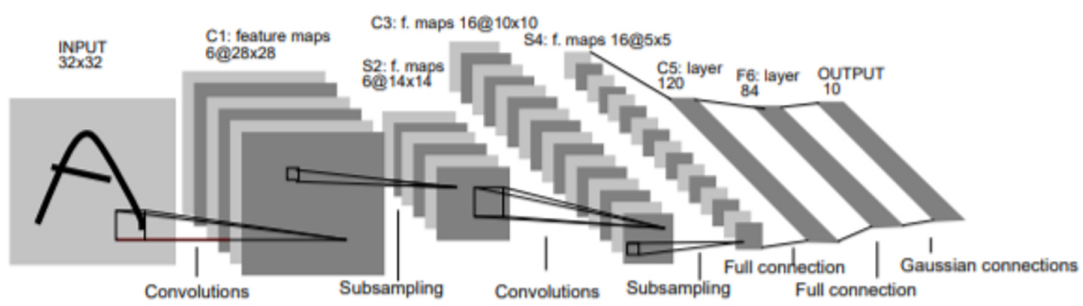

FIG. 2.3. Convolutional Neural Network [14]

and neural networks output, to achieve this it would repeatedly adjust weights of neurons. The procedure introduced was very different from the perceptron convergence method proposed earlier. They also introduced the concept of hidden units [13].

Subsequently, in 1998, Yann LeCun along with three other researchers introduced Convolutional Neural Networks for object recognition. They combined different operation like convolution max-pooling to extract features from images with Neural networks. Their work laid foundation to create a real time object recognition algorithm which is being used in self driving vehicles [14].

Current self-driving cars use Deep Learning technique to recognize objects. In this multi-layered neural network is trained on millions of images of different objects, the trained AI model is used to make prediction on the live camera feed being captured by the cameras mounted on the self driving vehicles. The algorithm draws bounding box around the object which helps in calculating the distance of the object from the self driving vehicles. Lane detection is also done on the live feed using image processing technique [15].

2.3. GPS. GPS's (Global Positioning System) birth came in 1960s during the cold war between the US and the Soviet Union. There was competition between these two countries on who could develop the best technology. American military understood importance of the technology when the Soviet Union the world's first satellite "Sputnik". Back then they used this technology to track submarines [16].

However, later in 1983 when the Korean airplane veered off into restricted Soviet airspace, they shot it down mistaking it for spy plane. To prevent such incidents, then President of the Soviet Reagen decided to release GPS technology.

Furthermore, After the release the first consumer GPS would cost 3000 USD. With time the technology kept improving day by day the initial device ran on short lasting batteries wasn't accurate enough. Other technologies also improved and supported GPS, with the developed of mobile phones GPS become a worldwide popular technology [17].

Today the technology has improved to a great extent and is very reliable due to this the same technology is being used in self driving vehicles for localization, tracking and route deciding [18].

3. Methodology. Current state of the art self driving vehicles include cameras, LiDAR, radar, GPS, wheel odometry, and IMUs [19]. An algorithm finds best possible route to reach the selected destination and the GPS constantly tracks current location of the vehicle. The vehicle's cameras records surrounding continuously, and the recorded video is processed in real time to find if any object is present or coming towards the vehicle. The cameras are also responsible for detecting lanes on the road this is how the vehicle the maintains lane discipline. However, night-time or different weather conditions increases risk of misjudging the situation which can result in accident. This can also lead to people loose faith in the technology which is improving day by day. Furthermore, some countries could not implement self driving vehicles because of their bad road infrastructure [20].

Our approach on other hand takes advantage of the deep learning technology to recognize objects and indication signs and overcome the disadvantages using GPS and AR technologies. The proposed approach uses GPS to get location and moving direction of the vehicle. From its location information virtual object is added to the original video. This new rendered video is fed into deep learning algorithm for detection various objects like traffic signal, lane lines, etc. based on this detection vehicle makes decision to which direction to steer [21]. 


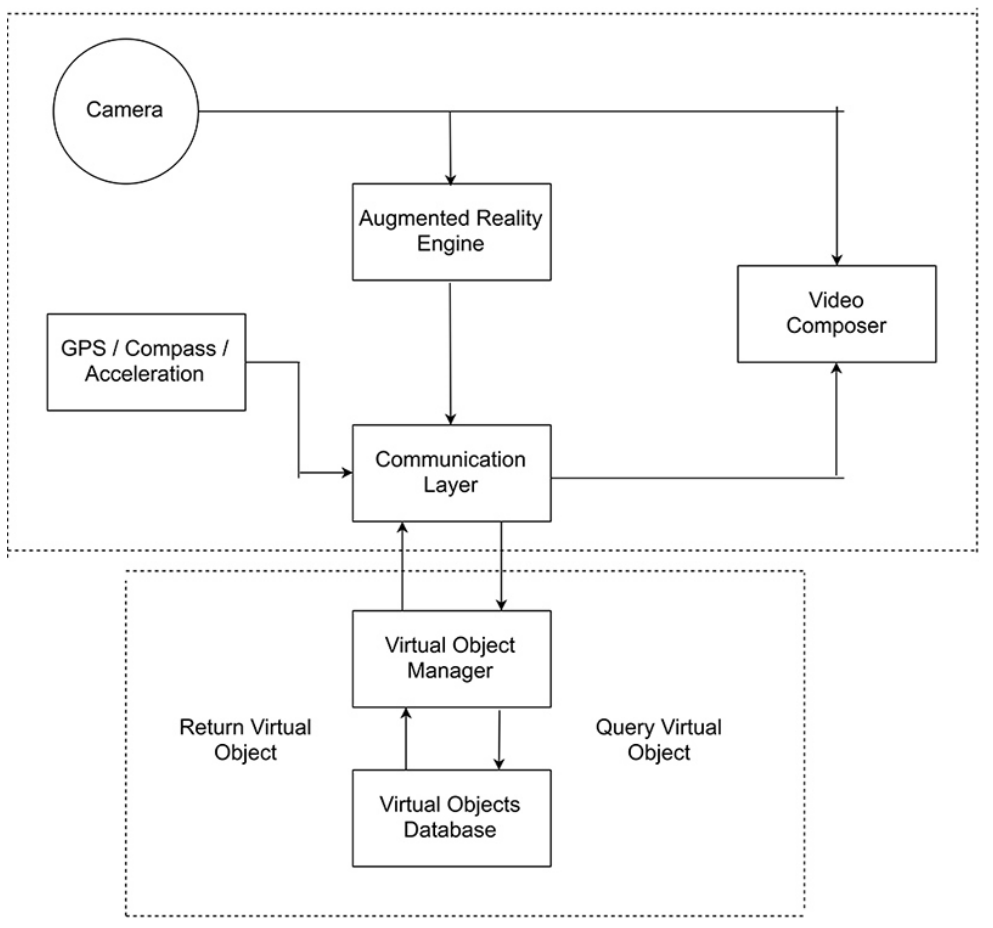

FIG. 3.1. AR Server Platform Architecture

Proposed ARIM algorithm process flow:

1. Getting current location of the vehicle using GPS.

2. Send the location of the vehicle to AR engine.

3. Based on location of the vehicle the virtual object manager makes request to Virtual object database.

4. Virtual object manager responds with the requested artificial object.

5. Video composer combines the graphical object and the original video captured by camera.

6. The new composed video is fed into the deep learning algorithm.

AR has two main components in it: Scene Generator and Tracking System. Scene generator is about showing the three-dimensional virtual object in the real-life environment. Rendering those virtual objects can be done to various levels depending on the requirement but simple graphics can also serve the purpose. Tracking system is about tracking the location of the user, the scene generator also depends on this as it has to modify the scene visuals depending on the perception of the user. Indoor navigation takes help of Bluetooth or local wi-fi devices to track current location of the person and based on that it shows indication on the phone to the destination.

The Virtual Object Manager and Virtual Object Database are two components of the system which work remotely. Communication layers manages the communication between the remote place and the actual place from where the request is made. GPS, compass and Accelerometer are one the most important components as the provide the information about location orientation of the device. Video composer composes the actual video with augmented virtual object [22].

The database of virtual objects is kept at a remote location and the Virtual Object Manager manages them [23]. The signs and lane lines are created in virtual navigational environment where will be able to see various signs while moving around which will guide us towards the destination.

The AR environment is created using FME AR software is explained in [24] the preparation process is described below:

1. First step is to take measurements of the place where the artificial objects are to be augmented.

2. Create an 3D model of the measurements on a 1:1 scale. 


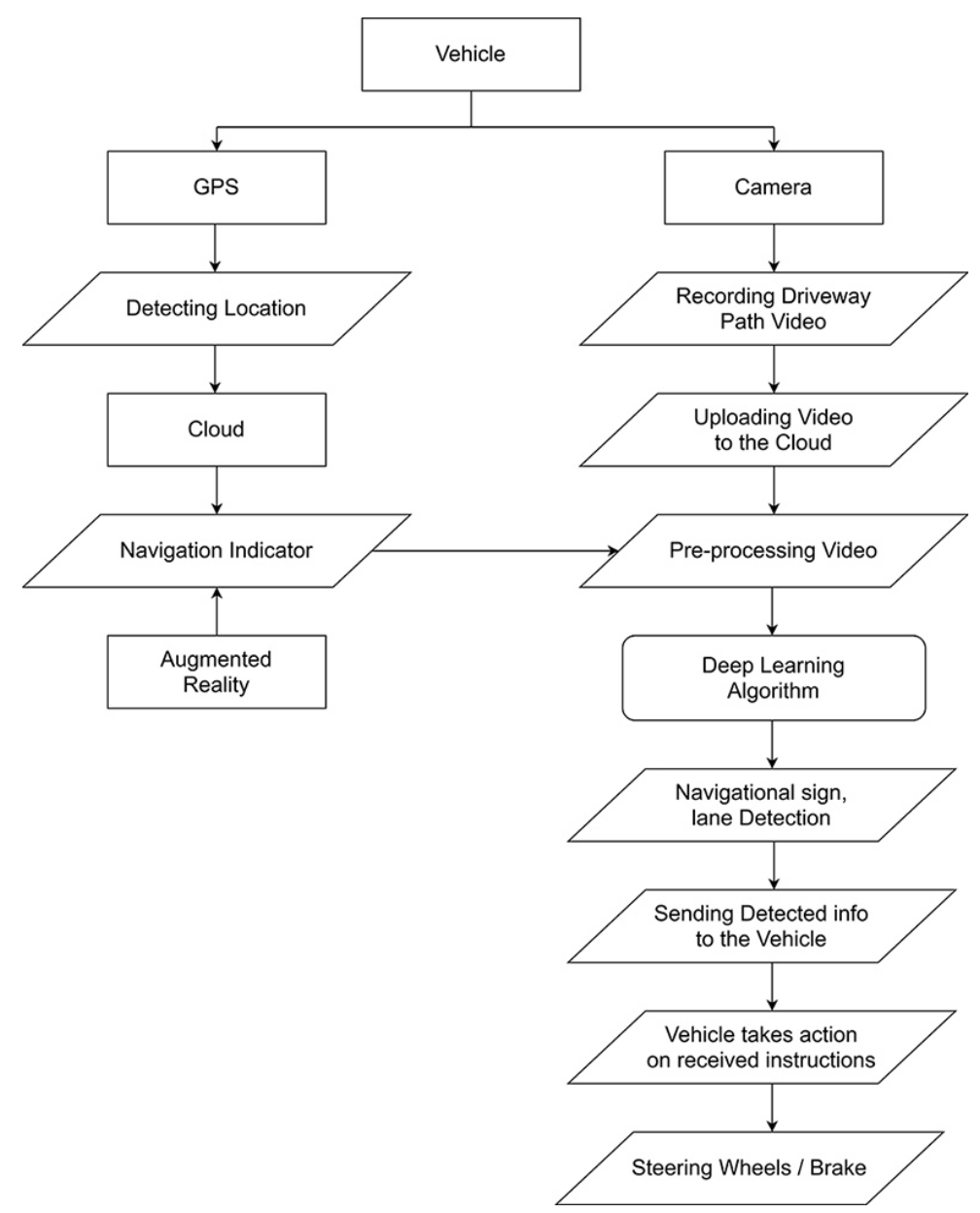

FIG. 3.2. ARIM Process Flow

3. Draw a rectangle of the A4 sheet size somewhere in the corner. The sheet will act as an anchor.

4. Now convert the model to .fmear using the FME software. Bring the file to the phone and open it in the FME AR app.

5. Place a real sheet of A4 size paper on the exact same location where you drew the virtual sheet of paper in the model.

6. Match the two sheets of paper by scaling, offsetting and rotating the model on the screen.

Now the FME AR application installed in the phone shows the signs and screen is shared to the cloud source where it is analysed for detection [25].

The approach is tested on a small self driving vehicles model created using Arduino microprocessor [26]. The model has a smartphone fixed on it [27]. The smartphone is fixed in a way that its back camera faced the travelling path. The smartphone is connected to a laptop over wi-fi network and the laptop is connected to the Arduino over Bluetooth network [28].

The smartphone records video of the travelling path and sends it to the laptop over wi-fi network. Here the laptop plays the part of the cloud server [29]. Laptop processes the video and feeds it into the deep learning algorithm to detect lanes, signs and objects [30]. Based on the detection later on the algorithm calculates the steering angle required to keep vehicle model in the lane and follow indications [31]. The calculated angle is then sent to the Arduino over Bluetooth, Arduino then takes action based on the received information [32]. 


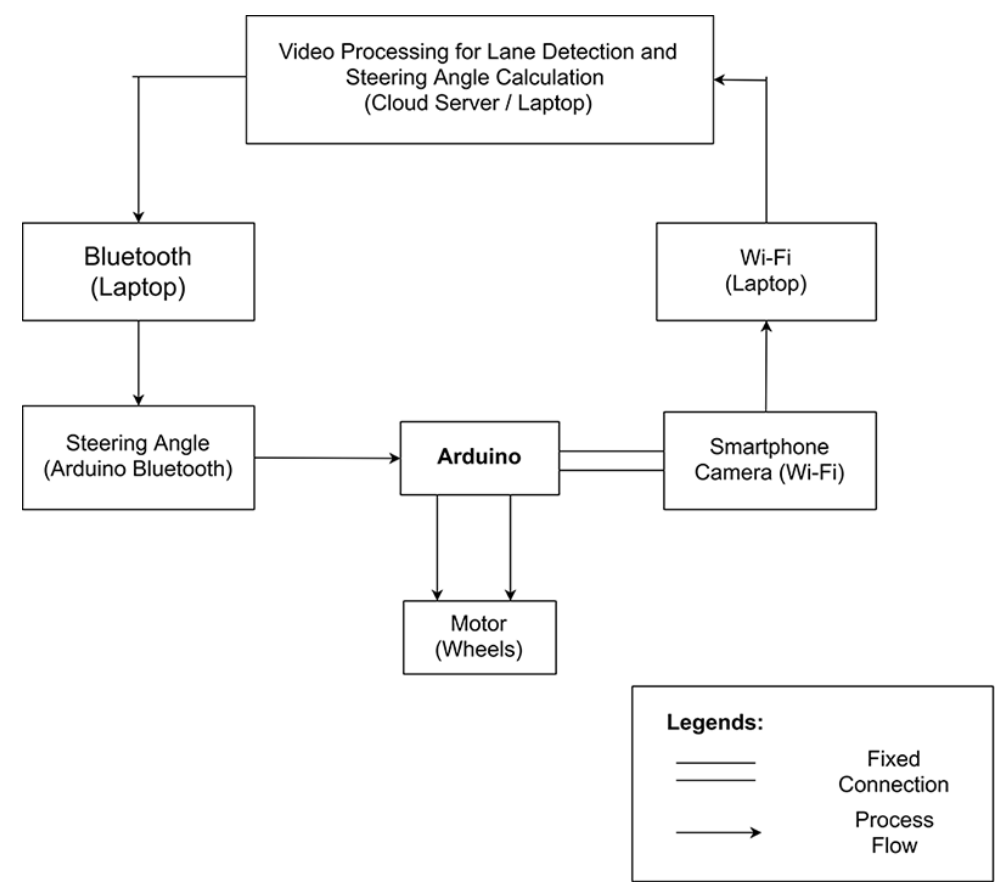

FIG. 3.3. Arduino Autonomous Vehicle Model Architecture

4. Results and Discussion. The most important work of detecting a sign or object is performed by the deep learning algorithm [33]. To test the approach, a deep neural network with 7 layers (1 Input layer, 5 hidden layers and an output layer) was trained. The hidden layers consist of 3 pairs of convolutional and max pooling layers and 2 fully connected layer [34]. The output layer makes prediction using SoftMax function [35].

The dataset used for the training consists of 51839 colour images of size 32x32. The dataset was split into 3 parts train, test and validation with 34799 images in train, 4410 images in validation and 12630 images for testing. The images are of 43 different classes. The classes included different road traffic signs like stop, right turn, one way etc. After training, the model achieved $93 \%$ accuracy on the test data [36].

To test the efficiency of the approach [38], the trained model was later used for prediction on traditional method and the AR method and results are compared [37]. Comparing the average precision on precision vs recall plot showed improved results with the proposed AR approach. To compare results of traditional and
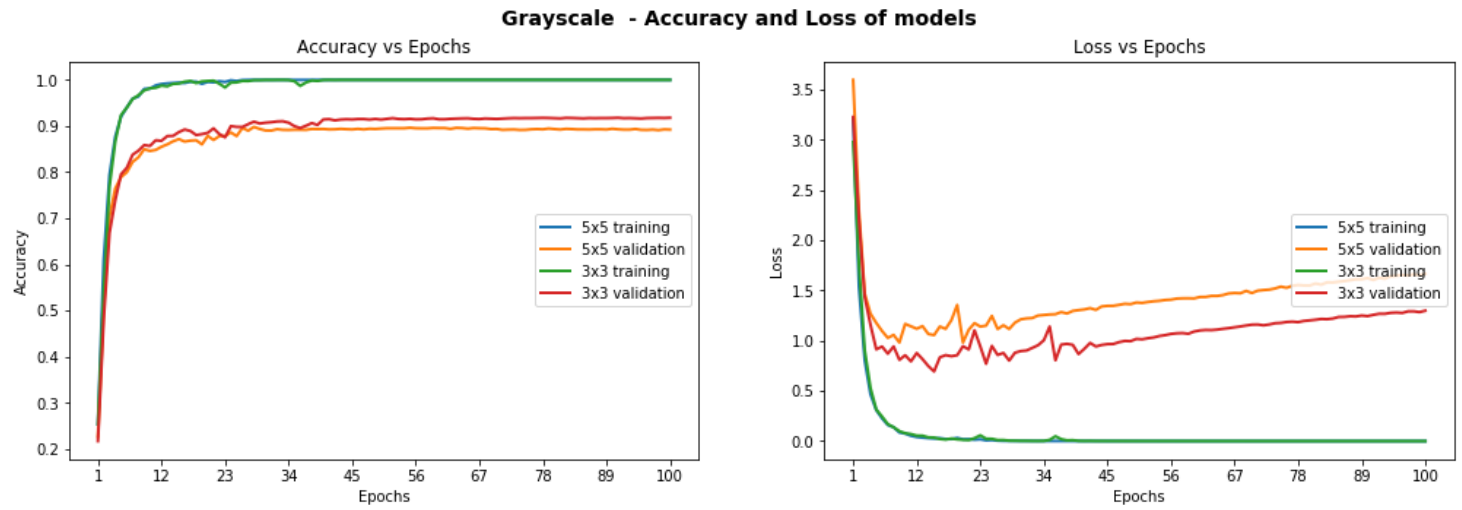

Fig. 4.1. Model Training Performance 

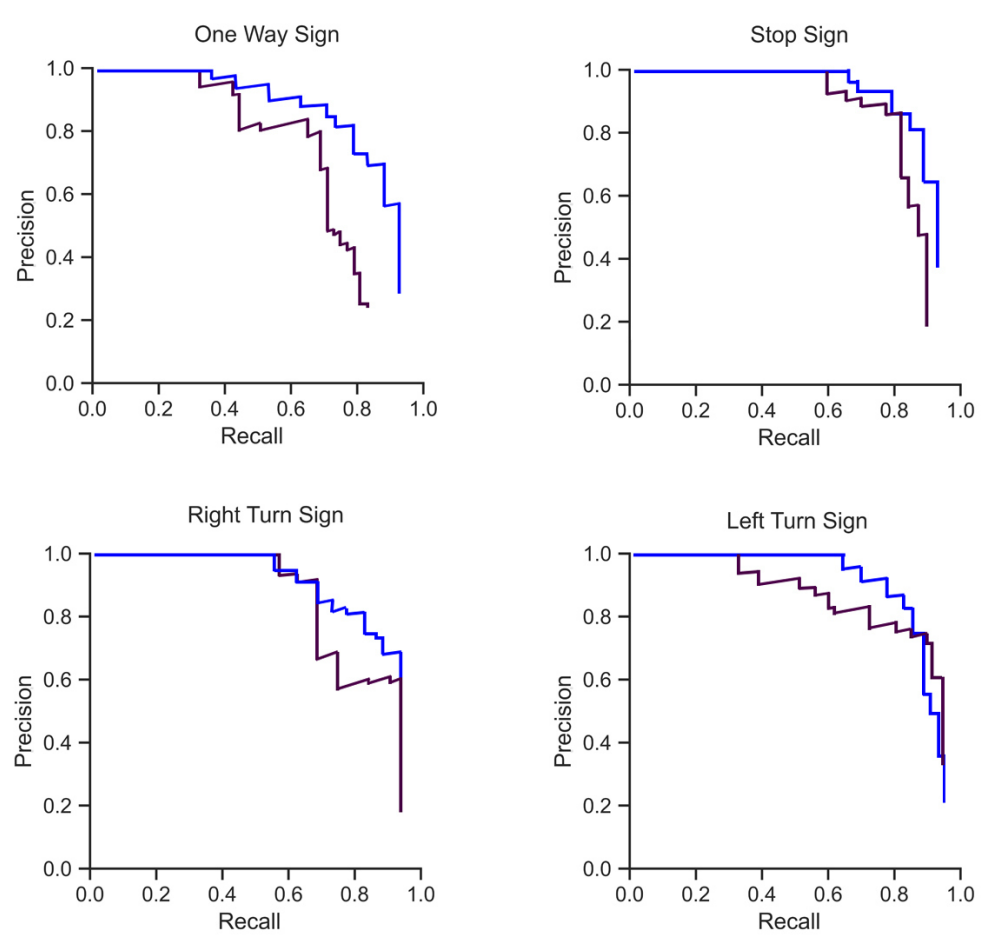

FIG. 4.2. Average precision comparison between traditional approach and AR approach

proposed approach, testing was performed on four different classes i.e. Stop Sign, One Way Sign, Right Turn Sign and Left Turn Sign [39].

Self driving vehicles don't just classify objects in a frame but also find their exact location. The algorithm draws bounding box around them for visualization. Performance of classification can be evaluated using confusing matrix but to calculate accuracy of an object detection algorithm mean average precision score is used. For better comprehension the scores are plot on Precision vs Recall graph. Below graphs show the performance of proposed approach comparing with traditional approach on precision vs recall plot [40].

In the graph the Magenta line indicates the traditional method and Blue line denotes proposed AR approach. The AR approach outperformed the traditional method with clear results. The ability of the traditional algorithm to detect a sign in a picture depends on how well it is visible in the picture. However, in the proposed approach signs are always present in a well accessible position making it easy to detect this was proved and validated by the results obtained.

5. Conclusion and Future Scope. The proposed algorithm is supposed to be used in self driving vehicles where safety of the passengers is of huge concern, hence it is important that the approach is properly tested. Testing the proposed algorithm on the real self driving vehicles was difficult, the test results presented in this paper was obtained by testing the approach in a local area map but the real self driving vehicles work on global map hence it is important to test the approach on a real self driving vehicle. However, it is expected that testing it locally with Bluetooth tracking was less accurate as compared to GPS hence the tracking will be better in the real self driving vehicles due to GPS and it can be integrated in a universal map like Google Maps.

The Arduino robot imitated role of self driving vehicles, the trained deep learning test model performed well on detecting the AR artificial navigational signs. As the objects were clear compared to real signs it was easier for the algorithm to detect. Using raspberry pi instead of Arduino could improve test results significantly and make the testing process seamless. Arduino's computational power is less compared to Raspberry pi, because 
of less computaional power the Arduino used in the testing has caused lag in responding to the instructions. In future work, Raspberry pi can be used to improve results [41].

The proposed work aims to lay a brick propose combining to technologies to promote the use of selfdriving by improving its safety and reliability. However, there is huge future scope to improve the system. One problem that was encountered during the testing was the artificial objects created using AR were hiding important surrounding details. The detail in real self driving vehicles can be any human or moving object which should be detected to avoid an accident. This problem can be resolved in future improvements of the algorithm. The AR system used for testing was not up to the mark as there was latency in between Bluetooth and Wi-Fi networks. By tunnelling the AR research approach for use in the self-driving vehicle can produce much better results.

\section{REFERENCES}

[1] World Health Organization, Global status report on road safety, Inj. Prev., 2015.

[2] KPMG, Autonomous Vehicles Readiness Index, Auton. Veh. Readiness Index, 2018.

[3] A. Teichman and S. Thrun, Practical object recognition in autonomous driving and beyond Proceedings of IEEE Workshop on Advanced Robotics and its Social Impacts, ARSO, 2011.

[4] G. National Crime Records Bureau, National Crime Records Bureau, Crime In India Reports from the Year 2002 to 2016 Crime In India Report, National Crime Records Bureau. 2016.

[5] M. Billinghurst, A. Clark, And G. LeE, A survey of augmented reality Foundations and Trends in Human-Computer Interaction. 2014

[6] H. Kato, Introduction to Augmented Reality Kyokai Joho Imeji Zasshi, Journal Inst. Image Inf. Telev. Eng., 2012.

[7] T. P. Caudell And D. W. Mizell, Augmented reality: an application of heads-up display technology to manual manufacturing processes 2003.

[8] B. Thomas et AL., ARQuake: an outdoor/indoor augmented reality first person application Int. Symp. Wearable Comput. Dig. Pap., 2000.

[9] W. Narzt et Al., Augmented reality navigation systems Univers. Access Inf. Soc., 2006.

[10] W. S. McCulloch And W. Pitts, A logical calculus of the ideas immanent in nervous activity Bull. Math. Biophys., 1943.

[11] F. Rosenblatt, The perceptron: A probabilistic model for information storage and organization in the brain Psychol. Rev., 1958.

[12] M. Minsky And S. PAPert, Perceptron: an introduction to computational geometry MIT Press. Cambridge, Expand. Ed., 1969.

[13] D. E. Rumelhart, G. E. Hinton, and R. J. Williams, Learning representations by back-propagating errors Nature, 1986.

[14] Y. LeCun, P. Haffner, L. Bottou, And Y. Bengio, Object recognition with gradient-based learning Lecture Notes in Computer Science (including subseries Lecture Notes in Artificial Intelligence and Lecture Notes in Bioinformatics), 1999.

[15] A. AZIM AND O. AYCARD, Detection, classification and tracking of moving objects in a 3D environmentIEEE Intelligent Vehicles Symposium, Proceedings, 2012.

[16] M. J. Rycroft, Understanding GPS. Principles and applications J. Atmos. Solar-Terrestrial Phys., 1997.

[17] H. Sugimoto, Introduction to GPS Seimitsu Kogaku Kaishi/Journal of the Japan Society for Precision Engineering. 2006.

[18] W. Rahiman And Z. ZAinal, An overview of development GPS navigation for autonomous car Proceedings of the 2013 IEEE 8th Conference on Industrial Electronics and Applications, ICIEA 2013,

[19] A. Kumar, M. Mukherjee, and P. Mukhopadhyay, Self Driving Car Advances in Intelligent Systems and Computing, 2020.

[20] G. H. Lee, F. FAundorfer, AND M. Pollefeys, Motion estimation for self-driving cars with a generalized camera in Proceedings of the IEEE Computer Society Conference on Computer Vision and Pattern Recognition, 2013.

[21] A. Y. C. Nee, S. K. Ong, G. Chryssolouris, And D. Mourtzis, Augmented reality applications in design and manufacturing CIRP Ann. - Manuf. Technol., 2012.

[22] O. Bimber And R. RAskar, Spatial augmented reality: Merging real and virtual worlds 2005.

[23] Q. Augmented and R. Lecture, ORB-SLAM: a Real-Time Accurate Monocular SLAM System IEEE Trans. Robot., 2015.

[24] Dmitri Bagh. (2020, March 01). Augmented Reality Indoor Mapping,

[25] Anand Nayyar, Bandana Mahapatra, D Le, G Suseendran, Virtual Reality (VR) and Augmented Reality (AR) technologies for tourism and hospitality industry International Journal of Engineering and Technology, 2018.

[26] M. MatiJevic And V. CvjetKovic, Overview of architectures with Arduino boards as building blocks for data acquisition and control systems in Proceedings of 2016 13th International Conference on Remote Engineering and Virtual Instrumentation, REV 2016.

[27] Vikram PURI, AnAND NAyYar, Real time smart home automation based on PIC microcontroller, Bluetooth and Android technology 3rd International Conference on Computing for Sustainable Global Development, 2016.

[28] A. Cotta, N. T. Devidas, And V. K. N. Ekoskar, Hc-05 Bluetooth Module Interfaced With ArduinoInt. J. Sci. Eng. Technol. Res., 2016.

[29] AnAND NAyyar, Vikram PuRI, Smart farming: IoT based smart sensors agriculture stick for live temperature and moisture monitoring using Arduino, cloud computing and solar technology The International Conference on Communication and Computing Systems, 2016. 
[30] Vikram Puri, Anand Nayyar, Dac-Nhuong Le, Handbook of Ardunio: Technical and Practice Scholars Press, 2017.

[31] RS Batth, A Nayyar, A NAGPal, Internet of robotic things: Driving intelligent robotics of future-concept, architecture, applications and technologies 4th International Conference on Computing Sciences (ICCS), 2018.

[32] A. K. JAIN, Working model of Self-driving car using Convolutional Neural Network, Raspberry Pi and Arduino Proceedings of the 2nd International Conference on Electronics, Communication and Aerospace Technology, ICECA 2018, 2018.

[33] Faisal Saeed, Anand Paul, P Karthigaikumar, Anand Nayyar, Convolutional neural network based early fire detection Multimedia Tools and Applications, 2019.

[34] C. Y. Lee, S. Xie, P. W. Gallagher, Z. Zhang, and Z. Tu, Deeply-supervised nets Journal of Machine Learning Research, 2015.

[35] N. KeTKaR, Deep Learning with Python. 2017

[36] S. Houben, J. Stallkamp, J. Salmen, M. Schlipsing, and C. Igel, Detection of traffic signs in real-world images: The German traffic sign detection benchmark Proceedings of the International Joint Conference on Neural Networks, 2013.

[37] Jafar Alzubi, Anand Nayyar, Akshi Kumar, Machine learning from theory to algorithms: an overview Journal of physics: conference series, 2018.

38] Jonathan Hui, mAP (mean Average Precision) for Object Detection Jonathan Hui - Medium, Mar 72018.

[39] C. Caragea et AL., Mean Average Precisionin Encyclopedia of Database Systems, 2009.

[40] T. Zeugmann et AL., Precision and Recall Encyclopedia of Machine Learning, 2011.

[41] Anand Nayyar, Vikram Puri, Raspberry Pi A Small, Powerful, Cost Effective and Efficient Form Factor Computer: A Review International Journal of Advanced Research in Computer Science and Software Engineering.

Edited by: Anand Nayyar

Received: Apr 24, 2020

Accepted: May 30, 2020 